\title{
Connectivity of the Lifts of a Greedoid
}

\author{
Steven J. Tedford \\ Department of Mathematics and Computer Science \\ Franklin and Marshall College \\ PO Box 3003, Lancaster, PA 17604-3003 \\ steven.tedford@fandm. edu \\ Submitted: Jul 16, 2006; Accepted: Apr 30, 2007; Published: May 23, 2007 \\ Mathematics Subject Classification: 05C99, 05B99
}

\begin{abstract}
Recently, attempts were made to generalize the undirected branching greedoid to a greedoid whose feasible sets consist of sets of edges containing the root satisfying additional size restrictions. Although this definition does not always result in a greedoid, the lift of the undirected branching greedoid has the properties desired by the authors.

The $k$-th lift of a greedoid has sets whose nullity is at most $k$ in the original greedoid. We prove that if the greedoid is $n$-connected, then its lift is also $n$ connected. Additionally, for any cut-vertex $v$ and cut-edge $e$ of a graph $\Gamma$, let $C(v)$ be the component of $\Gamma \backslash v$ containing the root and $C(e)$ be the component of $\Gamma \backslash e$ containing the root. We prove that if the $k$-th lift of the undirected branching greedoid is 2-connected, then

$$
\begin{aligned}
& |E(C(v))|<|V(C(v))|+k-1 \text { and } \\
& |E(C(e))|>|E(\Gamma)|-k-2 .
\end{aligned}
$$

We also give examples indicating that no sufficient conditions for the $k$ th lift to be 2-connected exists similar to these necessary conditions.
\end{abstract}

\section{Introduction and Definitions}

The undirected branching greedoid of a rooted graph has, as its feasible sets, sets of edges whose induced subgraphs are trees which contain the root. In [3], Li, Neumann-Lara, and Rivera-Campo attempted to generalize this greedoid by defining the feasible sets of a new greedoid to be sets of edges whose induced graphs are connected, contain the root, are restricted in size, and whose maximal sets span the graph. Although their construction does not always form a greedoid, the iterated lift of the undirected branching greedoid has the properties that they were interested in. Since they only used the existence of a greedoid with these properties, the existence of the iterated lift implies that the remaining applicable results in [3] still hold. 
The lift of a greedoid has as its feasible sets, sets whose nullity in the original greedoid is at most one. We show that if the original greedoid is $n$-connected then the lift is also $n$-connected.

In addition to using this result to show that iterated lift of the undirected branching greedoid possesses the properties desired by Li, et. al., we determine necessary conditions for graphs to have a 2-connected $k$ th lift. Finally, we give examples which show that conditions similar to the necessary conditions can not be sufficient conditions. We begin by introducing the necessary definitions, all of which can be found in [2, Chapters 4 and 5].

A greedoid $G$ consists of a ground set $E=E(G)$ and a nonempty collection of subsets $\mathcal{F}(G)$, called the feasible sets of $G$, satisfying:

(G1) If $F \neq \emptyset \in \mathcal{F}(G)$, then there exists $e \in F$ such that $F \backslash e \in \mathcal{F}(G)$.

(G2) If $F, F^{\prime} \in \mathcal{F}(G)$ with $\left|F^{\prime}\right|>|F|$, then there exists $e \in F^{\prime} \backslash F$ such that $F \cup e \in \mathcal{F}(G)$.

The rank of $X \subseteq E$ is defined to be $r_{G}(X)=\max \{|F|: F \in \mathcal{F}(G), F \subseteq X\}$. A maximal feasible set $F$ is called a basis of $G$. For any $X \subseteq E$, a basis of $X$ is a maximal feasible set contained in $X$. The nullity of $X$ in $G, \operatorname{nul}_{G}(X)$, is defined by

$$
\operatorname{nul}_{G}(X)=|X|-r_{G}(X)
$$

Given a greedoid $G$, the lift of $G$, denoted $L(G)=(E(G), \mathcal{F}(L(G)))$, has feasible sets defined by

$$
\mathcal{F}(L(G))=\left\{F \subseteq E: \operatorname{nul}_{G}(F) \leq 1\right\} .
$$

It is easy to show that this defines a greedoid. In addition, we define the $k$-th iterated lift of a greedoid recursively. Specifically, define

$$
L^{k}(G)= \begin{cases}G & \text { if } k=0 \\ L\left(L^{k-1}(G)\right) & \text { if } k>0 .\end{cases}
$$

This is equivalent to defining

$$
\mathcal{F}\left(L^{k}(G)\right)=\left\{F \subseteq E: \operatorname{nul}_{G}(F) \leq k\right\}
$$

Finally, a greedoid is 2-connected if, for any feasible set $F$, there exists a set $A$ with $|A|=\min \left\{2, r_{G}(E)-r_{G}(F)\right\}$ such that for all subsets $A^{\prime} \subseteq A, F \cup A^{\prime} \in \mathcal{F}(G)$. In general, a greedoid is $n$-connected if, for all feasible subsets $F$, there exists a set $A$ with $|A|=\min \{n, r-|F|\}$ such that for all subsets $A^{\prime} \subseteq A, F \cup A^{\prime} \in \mathcal{F}(G)$.

\section{Connectivity of Lifts of Greedoids}

First we show that connectivity is preserved when passing from a greedoid to its lift.

Theorem 2.1. If $k \geq 0, n \geq 2$, and $L^{k}(G)$ is $n$-connected, then $L^{i}(G)$ is n-connected for all $i \geq k$. 
Proof. By the definition of $L^{k}(G)$, it is enough to show that if $G$ is $n$-connected, then $L(G)$ is $n$-connected. For $L(G)$ to be $n$-connected, it must be true that for all $F \in \mathcal{F}(L(G))$, there exists a set $S \subseteq E \backslash F$ such that $|S|=\min \left\{n, r_{L}(E)-|F|\right\}$ and for any $S^{\prime} \subseteq S$, $F \cup S^{\prime} \in \mathcal{F}(L(G))$.

Suppose $F \in \mathcal{F}(L(G))$. There are two cases to consider.

1. Suppose $\operatorname{nul}_{G}(F)=0$. This implies that $F \in \mathcal{F}(G)$ and since $G$ is $n$-connected, there exists a set $A \subseteq E \backslash F$ such that $|A|=\min \left\{n, r_{G}(E)-|F|\right\}$ and $F \cup A^{\prime} \in \mathcal{F}(G)$ for all $A^{\prime} \subseteq A$. If $|A|=n$ or $r_{G}(E)=r_{L}(E)$, then let $S=A$. Otherwise, there exists an element $x \in E \backslash(F \cup A)$ such that $\operatorname{nul}_{G}(F \cup x)=1$. Let $S=A \cup x$.

2. Suppose $\operatorname{nul}_{G}(F)=1$ and $B$ is a basis of $F$ in $G$. Since $G$ is $n$-connected, there exists $A \subseteq E \backslash F$ such that $|A|=\min \left\{n, r_{G}(E)-|B|\right\}$ and for all $A^{\prime} \subseteq A, B \cup A^{\prime} \in$ $\mathcal{F}(G)$. If $r_{L}(E)=r_{G}(E)+1$, then $|A|=\min \left\{n, r_{L}(E)-|F|\right\}$ and let $S=A$. Otherwise, $|A|=\min \left\{n, r_{L}(E)-|F|+1\right\}$. Let $S$ be any subset of $A$ such that $|S|=\min \left\{n, r_{L}(E)-|F|\right\}$.

In either case, there exists a set $S$ such that $|S|=\min \left\{n, r_{L}(E)-|F|\right\}$ and $F \cup S^{\prime} \in$ $\mathcal{F}(L(G))$ for all $S^{\prime} \subseteq S$. Therefore $L^{i}(G)$ is $n$-connected for all $i>k$.

\section{Lifts of Undirected Branching Greedoids}

In [3], Li, et. al. attempted to generalize the undirected branching greedoid of a rooted graph. Suppose that $\Gamma$ is a graph with $\rho \in V(\Gamma)$. The undirected branching greedoid of $\Gamma$ rooted at $\rho$ has $E(\Gamma)$ as its ground set and feasible sets $F$ consisting of trees containing $\rho$. This greedoid is denoted $G_{\rho}=\left(E(\Gamma), \mathcal{F}\left(\Gamma_{\rho}\right)\right)$. If $\Gamma$ is connected, then $r\left(G_{\rho}\right)=|V(\Gamma)|-1$.

For any $|V(\Gamma)|-1 \leq k \leq|E(\Gamma)| \mathrm{Li}$, et. al. defined a set $F \subseteq E$ to be feasible in $G_{k}\left(\Gamma_{\rho}\right)$ as follows:

1. $|F| \leq k$ and $F$ forms a connected set containing $\rho$.

2. If $|F|=k$ then $F$ spans $\Gamma$.

Unfortunately, this collection of subsets does not form the feasible sets of a greedoid in general. In particular, if $\Gamma$ is the following graph with root $\rho$,

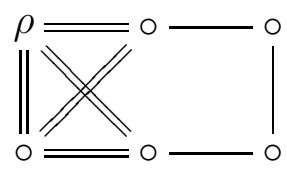

the set of edges marked by the double lines forms a feasible set in $G_{6}\left(\Gamma_{\rho}\right)$. However, since this set is not a basis and cannot be augmented to a cardinality 6 spanning set, $G_{6}\left(\Gamma_{\rho}\right)$ is not a greedoid.

The purpose of Li et. al. in introducing these $G_{k}$ 's was to show that a greedoid exists that has, for a 2-connected graph $\Gamma$, the following properties: 
L1 The greedoid is 2-connected.

L2 The bases of the greedoid consist of the spanning sets of edges consisting of exactly $k$ elements.

In [1] it was shown that for any $\rho \in V(\Gamma), \Gamma$ is 2 -connected if and only if $G_{\rho}$ is 2-connected. This, with Theorem 2.1 implies the following:

Corollary 3.1. If $\Gamma$ is 2-connected, then $L^{k}\left(G_{\rho}\right)$ is 2-connected for all $\rho \in V(\Gamma)$ and all $k \geq 0$.

This corollary, with the fact that the bases of $L^{k}\left(G_{\rho}\right)$ are exactly the sets of $k$ edges which span the graph, implies that $L^{k}\left(G_{\rho}\right)$ is a greedoid which satisfies both L1 and L2 above.

\section{Necessary Conditions for 2-connectivity of $L^{k}\left(G_{\rho}\right)$.}

In this section, we show that graphs whose $k$ th greedoid lift is 2-connected satisfy inequalities involving cut vertices and edges. For this purpose, if $v \in V(\Gamma)$ is a cut-vertex and $e \in E(\Gamma)$ is a cut-edge, define $C_{\rho}(v)$ to be the component of $\Gamma \backslash v$ which contains the root $\rho$ and $C_{\rho}(e)$ to be the component of $\Gamma \backslash e$ which contains the root $\rho$. For any $F \subseteq E(\Gamma)$, let $\Gamma_{F}$ be the subgraph of $\Gamma$ induced by the vertices of the graph induced by $F$. With this definition, we obtain the following necessary conditions for graphs whose $k$ th lift is 2-connected.

Theorem 4.1. Given a rooted graph $\Gamma_{\rho}$, if $L^{k}\left(G_{\rho}\right)$ is two connected then for every cutvertex $v$ and every cut-edge $e$,

$$
\begin{aligned}
\left|E\left(C_{\rho}(v)\right)\right| & <\left|V\left(C_{\rho}(v)\right)\right|+k-1 \text { and } \\
\left|E\left(C_{\rho}(e)\right)\right|>|E|-k-2 . & >
\end{aligned}
$$

Proof. First assume there exists a cut-vertex $v$ such that (1) does not hold. We can assume that there exist two edges $e_{1}$ and $e_{2}$ from $C_{\rho}(v)$ to $v$. Otherwise, there exists one cut-edge from $v$ to $C_{\rho}(v)$, and we can use the other vertex incident to the cut-edge. Let $B \subseteq E\left(C_{\rho}(v)\right)$ with $|B|=\left|V\left(C_{\rho}(v)\right)\right|+k-1$ which spans $C_{\rho}(v)$. This implies that $\operatorname{nul}_{G}(B)=k$, which implies $B, B \cup e_{1}, B \cup e_{2} \in \mathcal{F}\left(L^{k}\left(G_{\rho}\right)\right)$. However $B \cup\left\{e_{1}, e_{2}\right\} \notin$ $\mathcal{F}\left(L^{k}\left(G_{\rho}\right)\right)$. Any other edge would also increase the nullity of $B$. This implies that $L^{k}\left(G_{\rho}\right)$ is not 2-connected.

Next assume that there exists a cut-edge $e$ such that (2) does not hold. Suppose there exists a $B \subseteq E\left(C_{\rho}(e)\right)$ which spans $C_{\rho}(e)$ and has $|B|=\left|V\left(C_{\rho}(e)\right)\right|-1$. Since $|E|-\left|\left(E\left(C_{\rho}(e)\right) \cup e\right)\right| \geq k+1$, there exists a subset $X \subseteq E \backslash E\left(C_{\rho}(e)\right)$ which is connected, contains an edge adjacent to $e$ and has $|X|=k-1$. If $F=B \cup X$, $\operatorname{nul}_{G_{\rho}}(F)=k$; thus $F \in \mathcal{F}\left(L^{k}\left(G_{\rho}\right)\right)$. Furthermore there exists an edge $f \in E \backslash\left(E\left(C_{\rho}(e)\right) \cup(X \cup e)\right)$. Since $\operatorname{nul}_{G}(F \cup\{e, f\}) \leq \operatorname{nul}_{G}(F), F \cup\{e, f\} \in \mathcal{F}\left(L^{k}\left(G_{\rho}\right)\right)$. By construction, for any $A \subseteq E \backslash F$ with $|A|=2$, there exists an $A^{\prime} \subseteq A$ such that $F \cup A^{\prime} \notin \mathcal{F}\left(L^{k}\left(G_{\rho}\right)\right)$. Therefore $L^{k}\left(G_{\rho}\right)$ is not 2-connected. 
From Theorem 4.1, we immediately obtain the following for graphs without cut-edges:

1. If $L\left(G_{\rho}\right)$ is 2-connected then $C_{\rho}(v)$ is a tree for every cut-vertex $v \neq \rho$

2. If $L^{2}\left(G_{\rho}\right)$ is 2-connected then $C_{\rho}(v)$ contains at most one cycle for every cut-vertex $v \neq \rho$.

Once necessary conditions have been found, the next natural question is whether they are also sufficient. Unfortunately, these conditions are not sufficient. In fact, conditions similar to these are not sufficient, as shown by the following example.

Example 4.2. We consider a class of rooted graphs $\Gamma_{m, n}$ where $m, n \in \mathbb{N}, m \geq 1, n \geq 4$. Define $V\left(\Gamma_{m, n}\right)=\left\{\rho, v_{1}, v_{2}, v_{3}\right\}$ and $E\left(\Gamma_{m, n}\right)=\left\{a_{1}, \ldots, a_{m+1}\right\} \cup\{e\} \cup\left\{b_{1}, \ldots, b_{n-2}\right\}$. Each $a_{i}$ is incident to vertices $\rho$ and $v_{1}, e$ is incident to vertices $v_{1}$ and $v_{2}$, and each $b_{j}$ is incident to vertices $v_{2}$ and $v_{3}$.

Both $v_{1}$ and $v_{2}$ are cut-vertices. Now $\left|E\left(C_{\rho}\left(v_{1}\right)\right)\right|=0<\left|V\left(C_{\rho}\left(v_{1}\right)\right)\right|+m$. Also, $\left|E\left(C_{\rho}\left(v_{2}\right)\right)\right|=m+1<\left|V\left(C_{\rho}\left(v_{2}\right)\right)\right|+m$. Furthermore, $e$ is a cut-edge. Also $\left|E\left(C_{\rho}(e)\right)\right|=$ $m+1>m+n-n=|E|-n$. Thus $\Gamma_{m, n}$ satisfies the inequalities

$$
\begin{aligned}
& \left|E\left(C_{\rho}(v)\right)\right|<\left|V\left(C_{\rho}(v)\right)\right|+m \text { and } \\
& \left|E\left(C_{\rho}(e)\right)\right|>|E|-n
\end{aligned}
$$

for any cut-vertex $v$ and any cut-edge $e$.

However, for any $k<m+n-2, L^{k}\left(G_{\rho}\right)$ is not 2-connected. Since $k<m+n-2$, there exists a set $F \subseteq E$ which satisfies: $|F|=k+1, e \notin F,\left|F \cap\left\{a_{1}, \ldots, a_{m+1}\right\}\right| \geq 1$, and $\left|F \cap\left\{b_{1}, \ldots, b_{n-2}\right\}\right|>1$.

Since $r_{G_{\rho}}(F)=1$, and $|F|=k+1, \operatorname{nul}_{G_{\rho}}(F)=k$. Thus $F \in \mathcal{F}\left(L^{k}\left(G_{\rho}\right)\right)$. Suppose $A=\left\{f_{1}, f_{2}\right\} \subseteq E \backslash F$. If $e \in A$, then $F \cup e \in \mathcal{F}\left(L^{k}\left(G_{\rho}\right)\right)$. However, for any other edge $f$, $\operatorname{nul}_{G_{\rho}}(F \cup f)=k+1$, therefore $F \cup f \notin \mathcal{F}\left(L^{k}\left(G_{\rho}\right)\right)$. Thus $L^{k}\left(G_{\rho}\right)$ is not 2-connected.

Thus there is a whole class of graphs which show that conditions similar to the necessary condition are not sufficient.

This discussion leaves open the question regarding higher connectivity in the lifts of undirected branching greedoids. Since the necessary conditions for 2-connectivity of the lifts of the greedoids relates to connectivity of the graph, it is reasonable that such conditions also exist for higher connectivity of both the greedoids and the graphs.

\section{References}

[1] B. Korte and L. Lovász, Basis graphs of greedoids and 2-connectivity. In: R.W. Cottle (ed.) Mathematical Programming: Essays in Honor of George P. Dantzig, Vol I. pp. 158-165. Math. Programming Study, No. 24, North-Holland, Amsterdam, 1985.

[2] B. Korte, L. Lovász, and R. Schrader, Greedoids, Springer-Verlag, New York, 1991.

[3] X. Li, V. Neumann-Lara, E. Rivera-Campo, Two approaches for the generalization of leaf edge exchange graphs on spanning trees to connected spanning $k$-edge subgraphs of a graph, Ars Combin. 75 (2005), 257-265. 